\title{
Properties of a new integral operator
}

\section{Roberta Bucur, Loriana Andrei and Daniel Breaz}

\begin{abstract}
In this paper, we derive sufficient conditions for the univalence, starlikeness, convexity and some other properties in the class $N(\rho)$, for a new integral operator defined on the space of normalized analytic functions in the open unit disk.
\end{abstract}

\section{Introduction}

Let $\mathcal{A}$ be the class of functions which are analytic in the open unit disk $U=\{z:|z|<1\}$ given by

$$
f(z)=z+\sum_{k=2}^{\infty} a_{k} z^{k}, \quad z \in U
$$

Consider $S$ the subclass of $\mathcal{A}$ consisting of univalent functions. We denote by $S^{*}(\alpha)$ the class of starlike univalent functions of order $\alpha(0 \leq \alpha<1)$,

$$
S^{*}(\alpha)=\left\{f \in \mathcal{A}: \operatorname{Re}\left[\frac{z f^{\prime}(z)}{f(z)}\right]>\alpha, z \in U\right\} .
$$

By $K(\alpha)$ we denote a subclass of $\mathcal{A}$ consisting of convex univalent functions of order $\alpha(0 \leq \alpha<1)$ defined as

$$
K(\alpha)=\left\{f \in \mathcal{A}: \operatorname{Re}\left[\frac{z f^{\prime \prime}(z)}{f^{\prime}(z)}+1\right]>\alpha, z \in U\right\} .
$$

Key Words: analytic, starlike, convex functions, integral operator.

2010 Mathematics Subject Classification: 30C45.

Received: 06.04.2015

Accepted: 18.05.2015 
Clearly, we have

(i) $S^{*}(0)=S^{*}$ the class of all starlike functions with respect to the origin;

(ii) $K(0)=K$ the class of all convex functions;

(iii) $K \subset S^{*} \subset S, \quad K(\alpha) \subset S^{*}(\alpha), \quad K(\alpha) \subset K$ and $S^{*}(\alpha) \subset S^{*}$.

A function $f \in \mathcal{A}$ is said to be in the class $R_{\lambda}$ if and only if

$\operatorname{Re}\left[f^{\prime}(z)\right]>\lambda$, for some $\lambda, 0 \leq \lambda<1$.

Recently, Frasin and Jahangiri [4] define the family $B(\mu, \lambda), \mu \geq 0,0 \leq \lambda<$ 1 consisting of functions $f \in \mathcal{A}$ satisfying the condition

$$
\left|f^{\prime}(z)\left[\frac{z}{f(z)}\right]^{\mu}-1\right|<1-\lambda,
$$

for all $z \in U$.

It is obvious that: (i) $B(0, \lambda)=R_{\lambda}$; (ii) $B(1, \lambda)=S^{*}(\lambda)$;

(iii) $B(2, \lambda)=B(\lambda)$ (see Frasin and Darus [5]);

(iv) $B(2,0)=S$ (see Ozaki and Nunokawa [3]).

Let $N(\rho)$ be the subclass of $\mathcal{A}$ that contains all the functions $f$ which satisfy the inequality

$$
\operatorname{Re}\left[\frac{z f^{\prime \prime}(z)}{f^{\prime}(z)}+1\right]<\rho, \quad \rho>1, z \in U .
$$

Uralegaddi, Ganigi and Sarangi in [11] and Owa and Srivastava in [7] introduced and studied the class $N(\rho)$.

In the present paper, we introduce a new integral operator

$$
J_{\alpha}: \mathcal{A x} \mathcal{A} \rightarrow \mathcal{A}
$$

defined by:

$$
J_{\alpha}(f, g)(z)=\int_{0}^{z}\left[\frac{e^{f(t)}}{g^{\prime}(t)}\right]^{\alpha} d t
$$

where parameter $\alpha$ is a complex number, with $\operatorname{Re} \alpha \geq 1$.

In this paper our purpose is to obtain univalence conditions, starlikeness properties, the order of convexity for the integral operator abovementioned and to show that the operator $J_{\alpha}(f, g)(z)$ is in the class $N(\rho)$, by using functions from the class $B(\mu, \lambda)$. Recently, various types of integral operators were studied by different authors (see [10, 2]), and some of them motivated us to come up with the integral operator defined in (1.3).

In the proof of our main results, we need to recall here the following: 
Theorem 1.1. (Becker [1]) If the function $f$ is regular in the unit disk $U$, $f(z)=z+a_{2} z^{2}+\ldots$ and

$$
\left(1-|z|^{2}\right) \cdot\left|\frac{z f^{\prime \prime}(z)}{f^{\prime}(z)}\right| \leq 1
$$

for all $z \in U$, then the function $f$ is univalent in $U$.

Lemma 1.1. (General Schwarz Lemma [6]) Let $f$ be regular function in the disk $U_{R}=\{z \in \mathbb{C}:|z|<R\}$ with $|f(z)|<M$, $M$ fixed. If $f$ has in $z=0$ one zero with multiply bigger than $m$, then

$$
|f(z)| \leq \frac{M}{R^{m}}|z|^{m}, \quad z \in U_{R}
$$

The equality case hold only if $f(z)=e^{i \theta} \frac{M}{R^{m}} z^{m}$, where $\theta$ is constant.

Lemma 1.2. [9] Let the functions $p$ and $q$ be analytic in $U$ with

$$
p(0)=q(0)=0,
$$

and let $\delta$ be a real number. If the function $q$ maps the unit disk $U$ onto a region which is starlike with respect to the origin, the inequality

$$
\operatorname{Re}\left[\frac{p^{\prime}(z)}{q^{\prime}(z)}\right]>\delta, \text { for all } z \in U
$$

implies that

$$
\operatorname{Re}\left[\frac{p(z)}{q(z)}\right]>\delta, \text { for all } z \in U
$$

\section{Main results}

The univalence condition for the operator $I_{\alpha}(f, g)$ defined in (1.3) is proved in the next theorem, by using the Becker univalence criterion.

Theorem 2.1. Let $\alpha$ be a complex number, with $\operatorname{Re} \alpha \geq 1, f \in B(\mu, \lambda)$ and $g \in \mathcal{A}$. Suppose also that positive real numbers $M(M \geq 1)$ and $N(N \geq 1)$ are so constrained that

$$
|f(z)|<M \text { and }\left|\frac{g^{\prime \prime}(z)}{g^{\prime}(z)}\right| \leq N, \quad z \in U .
$$

If

$$
|\alpha| \leq \frac{3 \sqrt{3}}{2\left[(2-\lambda) M^{\mu}+N\right]},
$$

then the function $J_{\alpha}(f, g)$ is in the class $S$. 
Proof. Let the function $h$ be defined by

$$
h(z):=J_{\alpha}(f, g)(z), \quad z \in U .
$$

Obviously $h$ is regular in $U$ and $h(0)=h^{\prime}(0)-1=0$. From (2.3) we obtain

$$
\frac{z h^{\prime \prime}(z)}{h^{\prime}(z)}=\alpha\left[z f^{\prime}(z)-\frac{z g^{\prime \prime}(z)}{g^{\prime}(z)}\right] \text {. }
$$

Hence, we get

$$
\begin{gathered}
\left(1-|z|^{2}\right) \cdot\left|\frac{z h^{\prime \prime}(z)}{h^{\prime}(z)}\right| \leq\left(1-|z|^{2}\right) \cdot|z| \cdot|\alpha|\left[\left|f^{\prime}(z)\right|+\left|\frac{g^{\prime \prime}(z)}{g^{\prime}(z)}\right|\right] \\
\leq\left(1-|z|^{2}\right) \cdot|z| \cdot|\alpha|\left[\left(\left|f^{\prime}(z)\left(\frac{z}{f(z)}\right)^{\mu}-1\right|+1\right)\left|\frac{f(z)}{z}\right|^{\mu}+\left|\frac{g^{\prime \prime}(z)}{g^{\prime}(z)}\right|\right] .
\end{gathered}
$$

By using the hypothesis of the theorem and applying the General Schwarz Lemma, we have

$$
\left(1-|z|^{2}\right) \cdot\left|\frac{z h^{\prime \prime}(z)}{h^{\prime}(z)}\right| \leq\left(1-|z|^{2}\right) \cdot|z| \cdot|\alpha|\left[(2-\lambda) M^{\mu}+N\right] .
$$

Considering the function

$$
\begin{gathered}
t:[0,1) \rightarrow R, \\
t(x)=x\left(1-x^{2}\right), x=|z|,
\end{gathered}
$$

we find that

$$
t(x) \leq \frac{2}{3 \sqrt{3}}, \text { for all } x \in[0,1) .
$$

From (2.7), (2.8) and (2.6) we obtain

$$
\left(1-|z|^{2}\right) \cdot\left|\frac{z h^{\prime \prime}(z)}{h^{\prime}(z)}\right| \leq \frac{2|\alpha|}{3 \sqrt{3}}\left[(2-\lambda) M^{\mu}+N\right] \leq 1 .
$$

Finally, by applying Theorem 1.1 in (2.9) we yield that the function $J_{\alpha}(f, g)$ is in the class $S$.

In the following theorem we give sufficient conditions such that the integral operator $J_{\alpha}(f, g) \in S^{*}$. 
Theorem 2.2. Let $\alpha$ be a complex number, with $\operatorname{Re} \alpha \geq 1, f \in B(\mu, \lambda)$ and $g \in \mathcal{A}$. Suppose also that positive real number $M(M \geq 1)$ is so constrained that

$$
|f(z)|<M \text { and }\left|\frac{z g^{\prime \prime}(z)}{g^{\prime}(z)}\right|<1, \quad z \in U .
$$

If

$$
|\alpha| \leq \frac{1}{(2-\lambda) M^{\mu}+1},
$$

then the function $J_{\alpha}(f, g)$ is in the class $S^{*}$.

Proof. For the function $h$ be given by (2.3) we obtain

$$
\frac{z h^{\prime}(z)}{h(z)}=\frac{\frac{z e^{\alpha f(z)}}{\left[g^{\prime}(z)\right]^{\alpha}}}{\int_{0}^{z}\left[\frac{e^{f(t)}}{g^{\prime}(t)}\right]^{\alpha} d t} .
$$

Setting

$$
p(z)=z h^{\prime}(z) \text { and } q(z)=h(z),
$$

we find that $p(0)=q(0)=0$, and $q$ satisfies the starlikeness condition of Lemma 1.2. Since,

$$
\frac{p^{\prime}(z)}{q^{\prime}(z)}=1+\alpha\left[z f^{\prime}(z)-\frac{z g^{\prime \prime}(z)}{g^{\prime}(z)}\right]
$$

we obtain

$$
\left|\frac{p^{\prime}(z)}{q^{\prime}(z)}-1\right| \leq \cdot|\alpha|\left[\left(\left|f^{\prime}(z)\left(\frac{z}{f(z)}\right)^{\mu}-1\right|+1\right) \frac{|f(z)|^{\mu}}{|z|^{\mu-1}}+\left|\frac{z g^{\prime \prime}(z)}{g^{\prime}(z)}\right|\right] \text {. }
$$

Also, since $|f(z)|<M, z \in U$, applying the Schwarz Lemma, we have

$$
\left|\frac{f(z)}{z}\right| \leq M, \text { for all } z \in U \text {. }
$$

By using the hypothesis of the Theorem and replacing (2.14) in inequation (2.13), we obtain

$\left|\frac{p^{\prime}(z)}{q^{\prime}(z)}-1\right| \leq|\alpha| \cdot\left[\left(\left|f^{\prime}(z)\left(\frac{z}{f(z)}\right)^{\mu}-1\right|+1\right) M^{\mu}|z|+1\right] \leq|\alpha|\left[1+(2-\lambda) \cdot M^{\mu}\right] \leq 1$.

Thus, we have

$$
\operatorname{Re}\left[\frac{p^{\prime}(z)}{q^{\prime}(z)}\right]>0, \quad z \in U
$$


and, applying Lemma 1.2, we find that

$$
\operatorname{Re}\left[\frac{p(z)}{q(z)}\right]>0, \quad z \in U .
$$

This completes the proof of the theorem.

Letting $\mu=1$ in Theorem 2.2, we have

Corollary 2.1. Let $\alpha$ be a complex number, with $\operatorname{Re} \alpha \geq 1, f \in S^{*}(\lambda)$ and $g \in \mathcal{A}$. Suppose also that positive real number $M, M \geq 1$ is so constrained that

$$
|f(z)|<M \text { and }\left|\frac{z g^{\prime \prime}(z)}{g^{\prime}(z)}\right|<1, \quad z \in U .
$$

If

$$
|\alpha| \leq \frac{1}{1+(2-\lambda) M}
$$

then the function $J_{\alpha}(f, g)$ is in the class $S^{*}$.

Letting $\lambda=0$ in Corollary 2.1, we obtain

Corollary 2.2. Let $\alpha$ be a complex number, with $\operatorname{Re} \alpha \geq 1, f \in S^{*}$ and $g \in \mathcal{A}$. Suppose also that positive real number $M, M \geq 1$ is so constrained that

$$
|f(z)|<M \text { and }\left|\frac{z g^{\prime \prime}(z)}{g^{\prime}(z)}\right|<1, \quad z \in U .
$$

If

$$
|\alpha| \leq \frac{1}{1+2 M}
$$

then the function $J_{\alpha}(f, g)$ is in the class $S^{*}$.

Theorem 2.3. Let $\alpha$ be a complex number, with $\operatorname{Re} \alpha \geq 1, f \in B(\mu, \lambda)$ and $g \in \mathcal{A}$. Suppose also that positive real numbers $M(M \geq 1)$ and $N(N \geq 1)$ are so constrained that

$$
|f(z)|<M \text { and }\left|\frac{g^{\prime \prime}(z)}{g^{\prime}(z)}\right|<N, \quad z \in U .
$$

Then the function $J_{\alpha}(f, g)$ is in the class $K(\delta)$, where

$$
\delta=1-|\alpha|\left[N+(2-\lambda) \cdot M^{\mu}\right] \text { and } 0<|\alpha|\left[N+(2-\lambda) \cdot M^{\mu}\right] \leq 1 .
$$


Proof. By letting the function $h$ defined in (2.3), from equation (2.18) we find that

$$
\begin{aligned}
\left|\frac{z h^{\prime \prime}(z)}{h^{\prime}(z)}\right| & \leq|z| \cdot|\alpha|\left[\left|f^{\prime}(z)\right|+\left|\frac{g^{\prime \prime}(z)}{g^{\prime}(z)}\right|\right] \\
& \leq|z| \cdot|\alpha|\left[\left(\left|f^{\prime}(z)\left(\frac{z}{f(z)}\right)^{\mu}-1\right|+1\right)\left|\frac{f(z)}{z}\right|^{\mu}+\left|\frac{g^{\prime \prime}(z)}{g^{\prime}(z)}\right|\right] .
\end{aligned}
$$

From the hypothesis and applying the Schwarz Lemma in inequation (2.17), we obtain

$$
\left|\frac{z h^{\prime \prime}(z)}{h^{\prime}(z)}\right| \leq|\alpha|\left[N+(2-\lambda) \cdot M^{\mu}\right]=1-\delta .
$$

This evidently completes the proof.

Letting $\mu=1$ in Theorem 2.3, we have

Corollary 2.3. Let $\alpha$ be a complex number, with $\operatorname{Re} \alpha \geq 1, f \in S^{*}(\lambda)$ and $g \in \mathcal{A}$. Suppose also that positive real numbers $M(M \geq 1)$ and $N(N \geq 1)$ are so constrained that

$$
|f(z)|<M \text { and }\left|\frac{g^{\prime \prime}(z)}{g^{\prime}(z)}\right|<N, \quad z \in U .
$$

Then the function $J_{\alpha}(f, g)$ is in the class $K(\delta)$, where

$$
\delta=1-|\alpha|[N+(2-\lambda) M] \text { and } 0<|\alpha|[N+(2-\lambda) M] \leq 1 .
$$

Letting $\delta=\lambda=0$ in Corollary 2.3, we obtain

Corollary 2.4. Let $\alpha$ be a complex number, with $\operatorname{Re} \alpha \geq 1, f \in S^{*}$ and $g \in \mathcal{A}$. Suppose also that positive real numbers $M(M \geq 1)$ and $N(N \geq 1)$ are so constrained that

$$
|f(z)|<M \text { and }\left|\frac{g^{\prime \prime}(z)}{g^{\prime}(z)}\right|<N, \quad z \in U .
$$

Then the function $J_{\alpha}(f, g)$ is in the class $K$, where

$$
|\alpha|=\frac{1}{2 M+N} .
$$


Theorem 2.4. Let the functions $f, g \in \mathcal{A}$, with $f$ in the class $B(\mu, \lambda), \mu \geq$ $0,0 \leq \lambda<1$, and $\alpha$ a complex number, with $\operatorname{Re} \alpha \geq 1$. If $|f(z)|<M$, for $M a$ positive real number, $M \geq 1, z \in U$ and $\left|\frac{g^{\prime \prime}(z)}{g^{\prime}(z)}\right|<1$, then the integral operator $J_{\alpha}(f, g)$ defined by (1.3) is in the class $N(\rho)$, where

$$
\rho=|\alpha|\left[1+(2-\lambda) M^{\mu}\right]+1 .
$$

Proof. From (2.4) we obtain that

$$
\frac{z J_{\alpha}^{\prime \prime}(f, g)(z)}{J_{\alpha}^{\prime}(f, g)(z)}=\alpha z\left[f^{\prime}(z)-\frac{g^{\prime \prime}(z)}{g^{\prime}(z)}\right]
$$

So,

$$
\begin{aligned}
\operatorname{Re}\left[\frac{z J_{\alpha}^{\prime \prime}(f, g)(z)}{J_{\alpha}^{\prime}(f, g)(z)}+1\right] & =\operatorname{Re}\left[\alpha z\left(f^{\prime}(z)-\frac{g^{\prime \prime}(z)}{g^{\prime}(z)}\right)+1\right] \\
& \leq|z| \cdot|\alpha|\left[\left|f^{\prime}(z)\right|+\left|\frac{g^{\prime \prime}(z)}{g^{\prime}(z)}\right|\right]+1 \\
& \leq|z| \cdot|\alpha|\left[\left|f^{\prime}(z)\left(\frac{z}{f(z)}\right)^{\mu}\right|\left|\frac{f(z)}{z}\right|^{\mu}+1\right]+1
\end{aligned}
$$

Since $f$ is in the class $B(\mu, \lambda),|f(z)|<M$, from General Schwarz Lemma and from (2.18), we find that

$$
\begin{aligned}
\operatorname{Re}\left[\frac{z J_{\alpha}^{\prime \prime}(f, g)(z)}{J_{\alpha}^{\prime}(f, g)(z)}+1\right] & <|\alpha|\left[1+\left(\left|f^{\prime}(z)\left(\frac{z}{f(z)}\right)^{\mu}-1\right|+1\right) M^{\mu}\right]+1 \\
& <|\alpha|\left[1+(2-\lambda) M^{\mu}\right]+1=\rho
\end{aligned}
$$

We yield that the function $J_{\alpha}(f, g)$ is in the class $N(\rho)$.

For $\mu=0$ in Theorem 2.4 we obtain:

Corollary 2.5. Let the functions $f, g \in \mathcal{A}$, with $f$ in the class $R_{\lambda}, 0 \leq \lambda<1$, and $\alpha$ a complex number, with $\operatorname{Re} \alpha \geq 1$. If $|f(z)|<M$, for $M$ a positive real number, $M \geq 1, z \in U$ and $\left|\frac{g^{\prime \prime}(z)}{g^{\prime}(z)}\right|<1$, then the integral operator $J_{\alpha}(f, g)$ defined by (1.3) is in the class $N(\rho)$, where $\rho=|\alpha|(3-\lambda)+1$. 


\section{References}

[1] J. Becker, Lownersche Differential gleichung und quasi-konform fortsetzbare schlichte funktionen, J. Reine Angew. Math., 255(1972), 23-43.

[2] A. Oprea, D. Breaz, Univalence conditions for a general integral operator, Analele Univ. Ovidius, seria Matematica, Vol.23(1)(2015), 213-224.

[3] S. Ozaki, M. Nunokawa, The Schwarzian derivative and univalent functions, Proc. Amer. Math. Soc., 33(2)(1972), 392-394.

[4] B.A. Frasin, J. Jahangiri, A new and comprehensive class of analytic functions, Analele Univ. Oradea, Fasc. Math. XV(2008), 59-62.

[5] B.A. Frasin, M. Darus, On certain analytic univalent functions, Int. J. Math. and Math. Sci., 25(5)(2001), 305-310.

[6] O. Mayer, The functions theory of one variable complex, Bucuresti, 1981.

[7] S. Owa, H. M. Srivastava, Some generalized convolution properties associated with certain subclasses of analytic functions, Journal of Inequalities in Pure and Applied Mathematics, 3, article 42 (2002), 1-27.

[8] V. Pescar, New univalence criteria for some integral operators, Stud. Univ. Babes-Bolyai Math. 59(2014), No. 2, 167-176.

[9] G.L. Ready, K.S. Padmanabhan, On analytic function with reference to the Bernardi integral operator, Bull. Austral. Math. Soc., 25(1982), 387396.

[10] N. Ularu, D. Breaz, Univalence criterion and convexity for an integral operator, Applied Math. Letters 25, 3 (2012), 658-661.

[11] A. Uralegaddi, M.D. Ganigi, S.M. Sarangi, Univalent functions with positive coefficients, Tamkang Journal of Mathematics 25, 3(1994), 225-230.

Roberta BUCUR,

Department of Mathematics,

University of Piteşti,

Târgul din Vale St., No.1, 110040 Piteşti, România.

Email: roberta_bucur@yahoo.com

Loriana ANDREI,

Department of Mathematics and Computer Science,

University of Oradea,

1 Universitatii St., 410087 Oradea, România.

Email: lori_andrei@yahoo.com 
Daniel BREAZ,

Department of Mathematics,

"1 Decembrie 1918" University of Alba Iulia,

N. Iorga St., No. 11-13, 510009 Alba Iulia, România.

Email: dbreaz@uab.ro 